Research Paper

\title{
Phthalate esters used as plasticizers in packed red blood cell storage bags may lead to progressive toxin exposure and the release of pro-inflammatory cytokines
}

Leonard T. Rael, ${ }^{1}$ Raphael Bar-Or, ${ }^{1}$ Daniel R. Ambruso, ${ }^{2}$ Charles W. Mains, ${ }^{3}$ Denetta S. Slone, ${ }^{4}$ Michael L. Craun ${ }^{5}$ and David Bar-Or $1,6, *$

${ }^{1}$ Swedish Medical Center; Trauma Research; Englewood, CO USA; ${ }^{2}$ Bonfils Blood Center; Denver, CO USA; ${ }^{3}$ St. Anthony Central Hospital; Trauma Services; Denver, CO USA; ${ }^{4}$ Swedish Medical Center; Trauma Services; Englewood, CO USA; ${ }^{5}$ Scott and White Hospital; Trauma Services; Temple, Texas USA; ${ }^{6}$ Swedish Medical Center; Emergency Department; Englewood, CO USA

Abbreviations: PE's, phthalate esters; PRBC, packed red blood cells; DEHP, di-(2-ethylhexyl) phthalate; MEHP, mono-(2-ethylhexyl) phthalate; HUVEC, human umbilical vein endothelial cells; IL-8, interleukin-8

Key words: packed red blood cells, plasticizers, inflammation, blood transfusion, endothelial cells, oxidative stress, trauma

Phthalate esters (PE's) are plasticizers used to soften PVC-based medical devices. PE's are the most abundant man-made pollutants and increase the risk of developing an allergic respiratory disease or a malignancy. The leaching of PE's in donated packed red blood cells (PRBC) during storage was assessed. PRBC transfusion bags containing CPD/AS-1 (ADSOL) buffer were analyzed. Samples were collected on storage day 1 and day 42. Two PE's, di-(2-ethylhexyl) phthalate (DEHP) and mono-(2-ethylhexyl) phthalate (MEHP), were measured by liquid chromatography coupled to mass spectrometry (LCMS). Interleukin-8 (IL-8) was measured by standard ELISA techniques. DEHP significantly increased from $34.3 \mu \mathrm{M}( \pm 20.0 \mathrm{SD})$ on day 1 to $433.2 \mu \mathrm{M}$ $( \pm 131.2 \mathrm{SD})$ on day 42 , a 12.6 -fold increase. Similarly, MEHP significantly increased from $3.7 \mu \mathrm{M}( \pm 2.8 \mathrm{SD})$ on day 1 to 74.0 $\mu \mathrm{M}( \pm 19.1 \mathrm{SD})$ on day 42 , a 20.2 -fold increase. Also, DEHP and MEHP increased the release of IL-8 from human umbilical vein endothelial cells (HUVEC). The transfusion of older units of PRBC could lead to an accumulation of PE's possibly resulting in inflammation and other effects. This accumulation could be exacerbated due to the decreased metabolism of PE's since trauma patients have a lower esterase activity, the enzymes responsible for metabolizing PE's. The effect of oxidative stress caused by PE's is discussed as a potential mechanism for increases in inflammation caused by older units of PRBC.

*Correspondence to: David Bar-Or; Swedish Medical Center; Trauma Research Laboratory; 501 E. Hampden Ave. Rm. 4-454; Englewood, Colorado 80113 USA; Tel.: 303.788.4089; Fax: 303.788.4064; Email: dbaror@dmibio.com

Submitted: 03/27/09; Revised: 03/31/09; Accepted: 04/01/09

Previously published online as an Oxidative Medicine and Cellular Longevity E-publication:

http://www.landesbioscience.com/journals/oximed/article/8608

\section{Introduction}

Phthalate esters are a group of plasticizers produced commercially to impart flexibility to an otherwise rigid polyvinylchloride (PVC). ${ }^{1} \mathrm{PVC}$ and phthalates are used extensively in the production of blood collection bags and other medical devices. In 2002, more than 18 billion pounds of phthalates were used in the manufacturing of plastics worldwide. ${ }^{1}$ In particular, DEHP, the most commonly used plasticizer, accounted for over $20 \%$ of the total phthalates produced annually. ${ }^{1}$ Consequently, phthalates are widely distributed in the ecosystem and are among the most abundant man-made environmental pollutants. Although human urinary excretion levels are usually below exposure limit values of the US EPA and other worldwide environmental agencies, the cumulative chronic exposure to all phthalates and possible synergistic endocrine effects need to be investigated. ${ }^{2}$

In the last 10 years, extensive laboratory studies have attempted to determine the impact of long-term phthalate exposure on human health. A metabolite of DEHP, MEHP, was shown to be carcinogenic by activating two nuclear transcription factors, PPAR $\alpha$ and PPAR $\gamma$, important to cell differentiation. ${ }^{3}$ Additionally, DEHP has been shown to be a developmental and reproductive toxicant suspected of having endocrine disrupting or modulating effects. ${ }^{4}$ The development of some allergic respiratory diseases has been attributed to DEHP and MEHP with the former causing an increase in $\operatorname{IgG}$ and the latter promoting increased levels of pro-inflammatory cytokines such as interleukin-6 (IL-6) and interleukin-8 (IL-8).., 6

Almost all toxicological studies of DEHP (Fig. 1A) and MEHP (Fig. 1B) only consider ingestion, inhalation and dermal exposure. These three exposure routes still have to undergo absorption to the bloodstream. In blood bags and other phthalate-containing medical devices, the absorption pathway is skipped due to the intravenous 
administration of blood bag contents. Therefore, we measured the amount of DEHP and MEHP in the supernatants of packed red blood cell (PRBC) bags collected at a blood donation center using liquid chromatography/mass spectrometry (LCMS) techniques. Phthalate levels were measured on day 1 post-donation and day 42 which is the expiration date for stored PRBC. Our results are discussed in relation to the deleterious health effects sometimes seen in the administration of older blood products. ${ }^{7}$ Also, a potential oxidative stress mechanism addressing the pro-inflammatory processes caused by DEHP and MEHP is proposed.

\section{Results}

Using DEHP and MEHP standards, the amount of each phthalate ester was measured in the day 1 and day 42 supernatants collected from PVC bags containing donated PRBC. DEHP (Fig. 2) and MEHP (Fig. 3) had retention times of 10.5 and 7.4 minutes, respectively. Dissolving stocks of the standards in acetonitrile and then diluting to working concentrations in 1X PBS did not significantly alter the retention times of either phthalate compared to the phthalate retention times observed in the sample supernatants. In addition to retention times, DEHP (Fig. 2) and MEHP (Fig. 3) were identified by their expected monoisotopic mass $\left(\mathrm{M}+\mathrm{H}^{+}\right)$of 391.28 and 279.16 , respectively. During the 42 day storage period, DEHP increased from $34.3 \mu \mathrm{M}$ $( \pm 20.0 \mathrm{SD})$ on day 1 to $433.2 \mu \mathrm{M}( \pm 131.2 \mathrm{SD})$ on day 42 , a 12.6-fold increase (Fig. 4A). This is visually evident when comparing the peak heights in the representative mass chromatograms for the day 1 sample (Fig. 2A) and day 42 sample (Fig. 2B). Similarly, MEHP levels increased from $3.7 \mu \mathrm{M}$ $( \pm 2.8 \mathrm{SD})$ to $74.0 \mu \mathrm{M}( \pm 19.1 \mathrm{SD})$, a 20.2 -fold increase (Fig. 4B). Again, the MEHP increase can be observed when comparing the peak heights in the representative mass chromatograms for the day 1 sample (Fig. 3A) and day 42 sample (Fig. 3B).

To assess the pro-inflammatory potential of the phthalate esters, HUVEC were dosed with nanomolar concentrations of MEHP and DEHP. At almost all dosage concentrations used, both MEHP and DEHP caused significant increases in IL-8 release (Fig. 5). With increasing MEHP concentrations, an increase in IL-8 release was observed. However, IL-8 release decreased as DEHP concentrations increased, but the amount of IL-8 release was still significantly higher than controls except for the $1,000 \mathrm{nM}$ concentration. A concentration of greater than 1,000 nM DEHP caused significant decreases in IL-8 release accompanied by significant decreases in HUVEC viability. For example, $100 \mu \mathrm{M}$ DEHP caused a $60 \%$ decrease in IL-8 release with a corresponding 50\% decrease in cell viability.

\section{Discussion}

Here, we show the leaching of the plasticizer DEHP and MEHP from polyvinyl chloride (PVC) bags containing donated PRBC collected at a local blood bank. The initial levels of DEHP $(34.3 \mu \mathrm{M})$ and MEHP $(3.7 \mu \mathrm{M})$ correspond well with a previously published work that measured DEHP and MEHP concentrations ranging from $25-50 \mu \mathrm{M}$ and 3-14 $\mu \mathrm{M}$, respectively, in red blood cell concentrates. ${ }^{8}$ This previous work also observed a five-fold

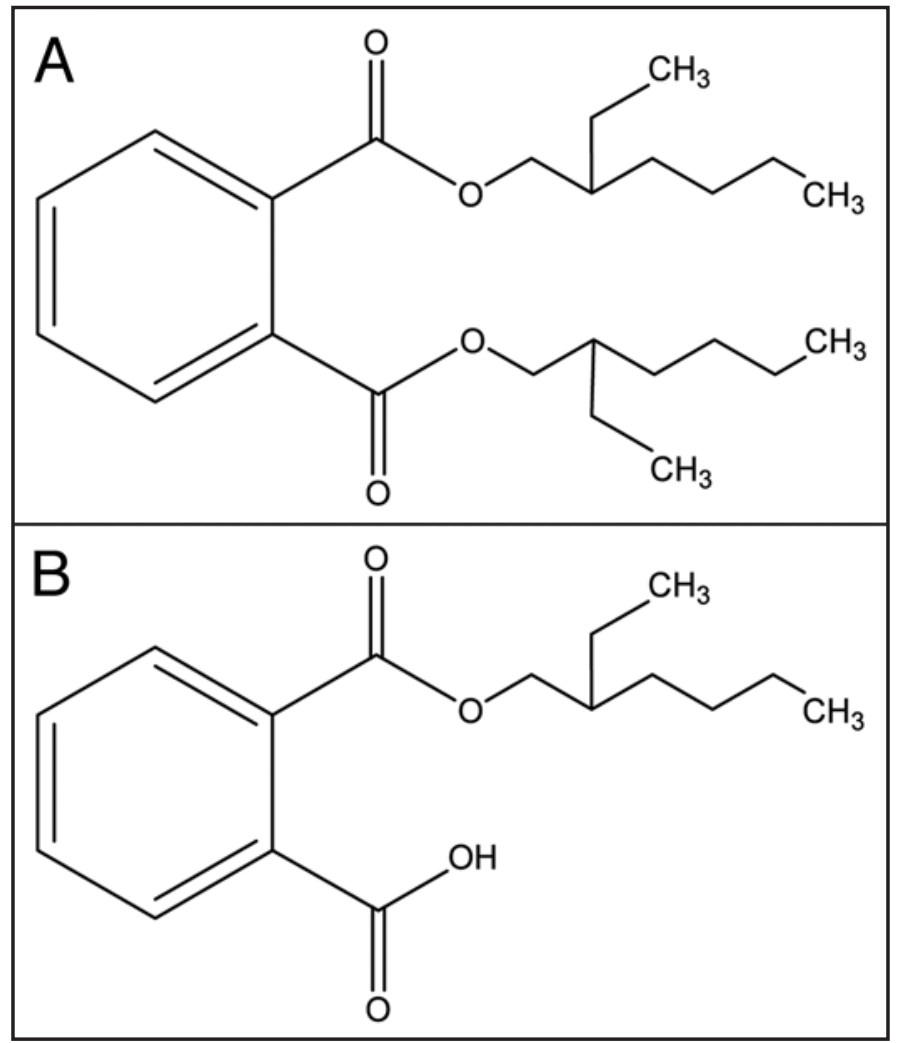

Figure 1. Chemical structure of (A) DEHP and (B) MEHP.

increase in DEHP levels over a 21-day storage period. ${ }^{8}$ In patients undergoing hemodialysis due to chronic renal failure, a high concentration of DEHP in blood was measured in these patients attributable to the leaching of DEHP from the hemodialysis tubing. ${ }^{9,10}$

In human plasma, DEHP is rapidly metabolized by ester hydrolysis to MEHP which is subsequently glucuronidated and excreted in urine. ${ }^{1,10}$ MEHP accumulation is of concern because MEHP is 20 times more toxic than DEHP in rats. ${ }^{11}$ Additionally, MEHP has a longer plasma half-life due to its ability to bind to serum proteins, specifically to human serum albumin. ${ }^{12,13}$ Due to its lipophilic characteristics, DEHP also exerts many biochemical effects such as decreasing its own metabolism by inhibiting serum arylesterase activity levels. ${ }^{14}$ Administering blood products in phthalate-containing bags, especially older blood products, to trauma patients could have deleterious results due to the already decreased arylesterase activity levels found in plasma collected from these particular patients. ${ }^{15}$

The effect of DEHP and MEHP on IL-8 release from HUVEC was assessed. At nanomolar concentrations, both phthalate esters significantly increased IL-8 release from HUVEC. The concentrations used in the cell culture experiment are comparable to the concentrations of the phthalate esters measured in the PRBC supernatants after accounting for dilution of the transfusion in the circulation. A typical PRBC unit contains about $200 \mathrm{~mL}$ of erythrocytes suspended in about $50 \mathrm{~mL}$ preservation fluid. ${ }^{16}$ 


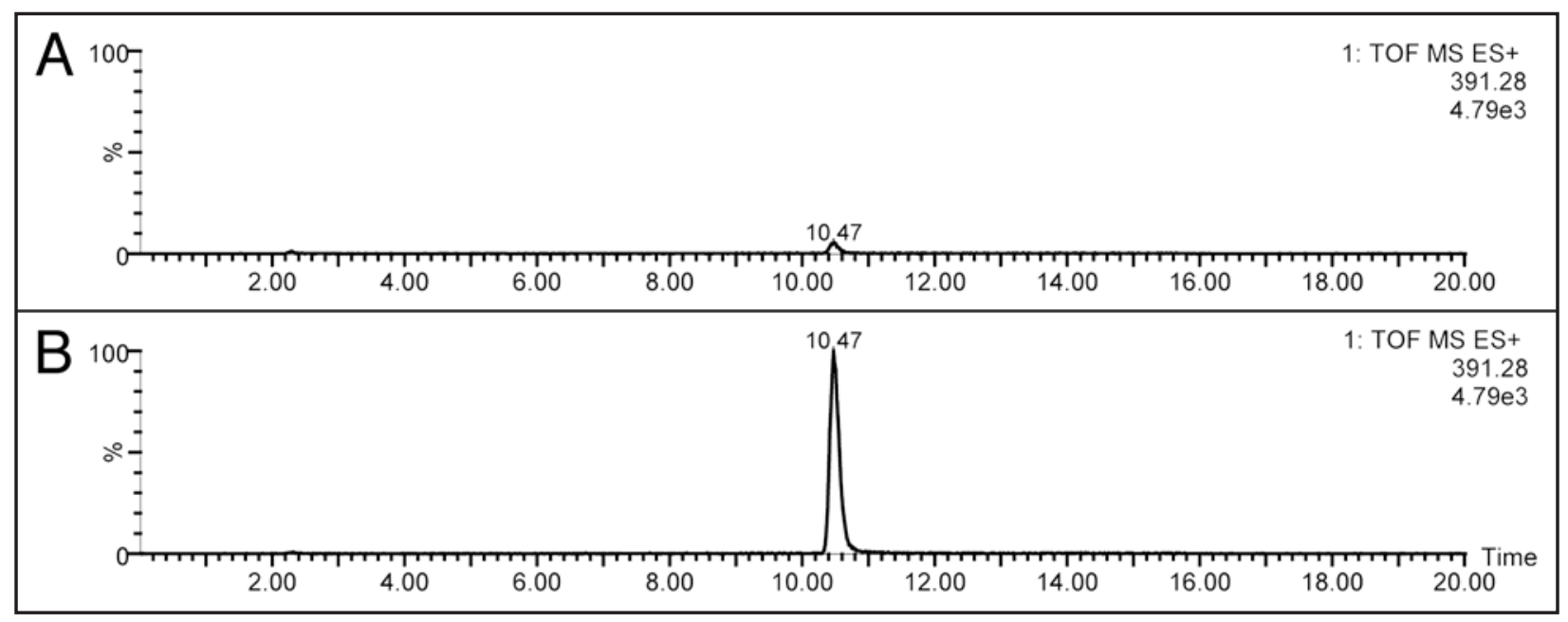

Figure 2. Representative mass chromatograms measuring DEHP detected in day 1 (A) and day 42 (B) supernatants collected from stored packed red blood cell (PRBC) units $(\mathrm{N}=10)$. DEHP $\left(\mathrm{RT}=10.5 \mathrm{~min},\left[\mathrm{M}+\mathrm{H}^{+}\right]=391.28\right)$ was detected using prepared standards (1-1,000 $\left.\mu M\right)$. For each run, $10 \mu \mathrm{L}$ of sample was injected onto a YMC-Pack Protein-RP HPLC column (Waters, Milford, MA) heated to $50^{\circ} \mathrm{C}$. A 20 minute linear gradient from 10 to $40 \%$ solvent B using water/0.1\% trifluoroacetic acid (solvent A) and acetonitrile/0.1\% trifluoroacetic acid (solvent B) was utilized with a flow rate of $1 \mathrm{~mL} / \mathrm{min}$.

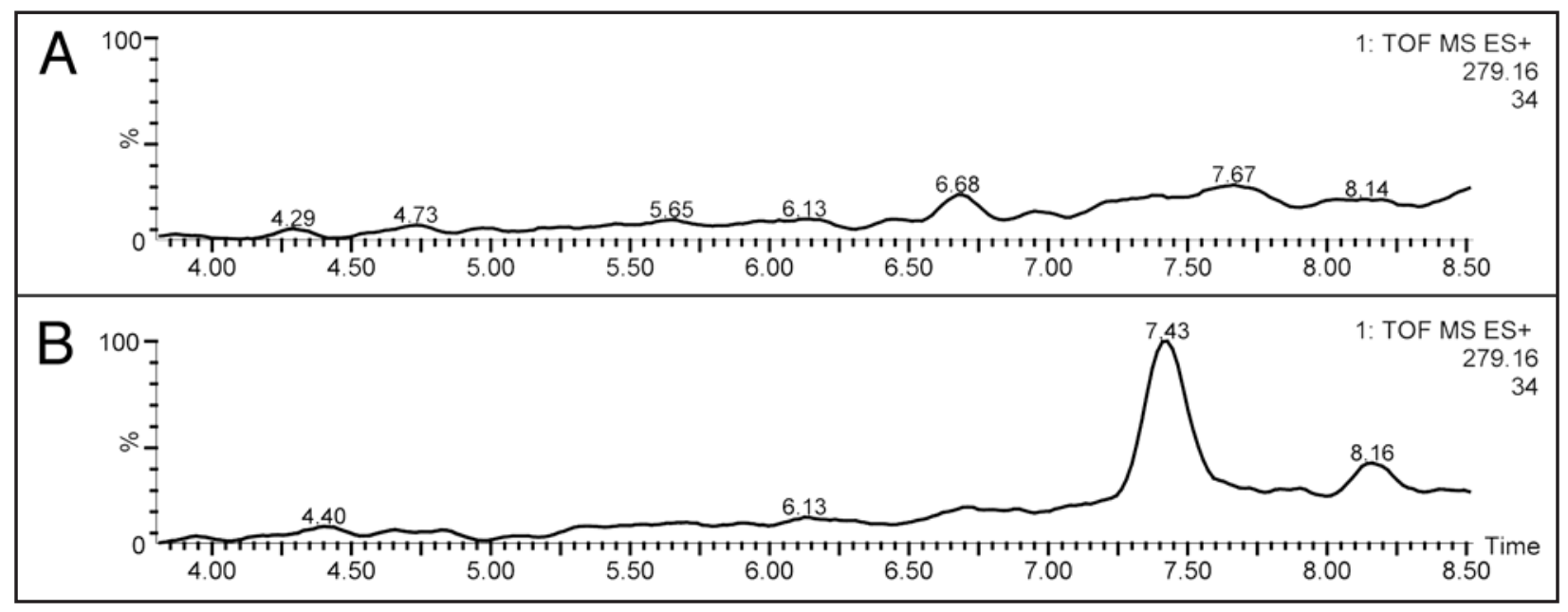

Figure 3. Representative mass chromatograms measuring MEHP detected in day 1 (A) and day 42 (B) supernatants collected from stored packed red blood cell (PRBC) units $(\mathrm{N}=10)$. MEHP $\left(\mathrm{RT}=7.4 \mathrm{~min},\left[\mathrm{M}+\mathrm{H}^{+}\right]=279.16\right)$ was detected using prepared standards $(1-100 \mu M)$. For each run, $10 \mu \mathrm{L}$ of sample was injected onto a YMC-Pack Protein-RP HPLC column (Waters, Milford, MA) heated to $50^{\circ} \mathrm{C}$. A 20 minute linear gradient from 10 to $40 \%$ solvent B using water $/ 0.1 \%$ trifluoroacetic acid (solvent A) and acetonitrile/0.1\% trifluoroacetic acid (solvent B) was utilized with a flow rate of $1 \mathrm{~mL} / \mathrm{min}$.

Since phthalate ester concentrations were only measured in the supernatants (i.e., preservation fluid) of PRBC units using our methodology, a 100 -fold dilution in a transfused patient can be expected assuming a total blood volume of 5 liters. Therefore, after dilution in the circulation, transfusion of 42-day-old blood translates to plasma levels of $740 \mathrm{nM} \mathrm{MEHP}$ and 4,330 nM DEHP per unit of PRBC transfused based on our findings.

The exact biochemical mechanism that causes the release of IL-8 from endothelial cells treated with DEHP or MEHP was not determined in our model. There is supporting evidence that oxidative stress caused by DEHP or MEHP exposure results in pro-inflammatory events such as IL-8 release. For example, exposure to DEHP causes significant increases in markers of oxidative stress in liver. ${ }^{17,18}$ Also, in urban adult populations, a direct correlation was observed between urinary oxidative stress biomarkers and the degree of MEHP exposure. ${ }^{19}$ In endothelial cells, oxidative stress induces the release of IL-8 in a dosedependent manner. ${ }^{20,21}$ Therefore, it is conceivable that DEHP and/or MEHP cause the release of pro-inflammatory mediators such as IL-8 via an oxidative stress mechanism. This theory is further supported by our observation that the presence of an oxidative environment increases during storage time in the same PRBC transfusion bags in which we measured DEHP and MEHP levels. ${ }^{22}$ 
Unfortunately, we did not measure DEHP and MEHP levels in the RBC pellet that could significantly contribute to the total amount of phthalate esters administered to a patient. Since this study was part of a larger study, only the supernatants from the PRBC units were saved for this study. Approximately $28 \%$ of available DEHP binds immediately to sites in both the membrane and cytosol fractions of erythrocytes. ${ }^{23}$ The contribution of DEHP bound to erythrocyte membranes to potential health hazards in transfused patients is currently unknown. If DEHP incorporates itself in the HUVEC membranes, then that could partially explain the decrease in viability seen at micromolar concentrations of DEHP.

Utilizing banked blood products in the critically ill is a common medical practice employed worldwide for obvious beneficial reasons. Infusion-related risk factors, such as transfusion-related acute lung injury (TRALI) and other inflammatory pathologies, increase proportionally with the age of the infused blood product. ${ }^{7}$ Here, we show an increase in the supernatant concentrations of the phthalates DEHP and MEHP in bags containing PRBC between storage day 1 and day 42. Due to our limited access to the storage bags, we were unable to monitor the accumulation of the phthalate esters on a daily basis. This would determine whether phthalate accumulation is a linear progression or reaches a critical point that allows for the exponential increase in phthalate concentrations. In the future, a clinical study correlating DEHP and/or MEHP plasma levels with the development of proinflammatory based pathologies in the critically ill could be interesting. Based on our study, we suggest that the transfusion of older units of PRBC in trauma patients could lead to an accumulation of DEHP and MEHP. Due to the known biochemical actions of DEHP and MEHP, this accumulation could possibly result in pro-inflammatory and other effects in transfused patients.

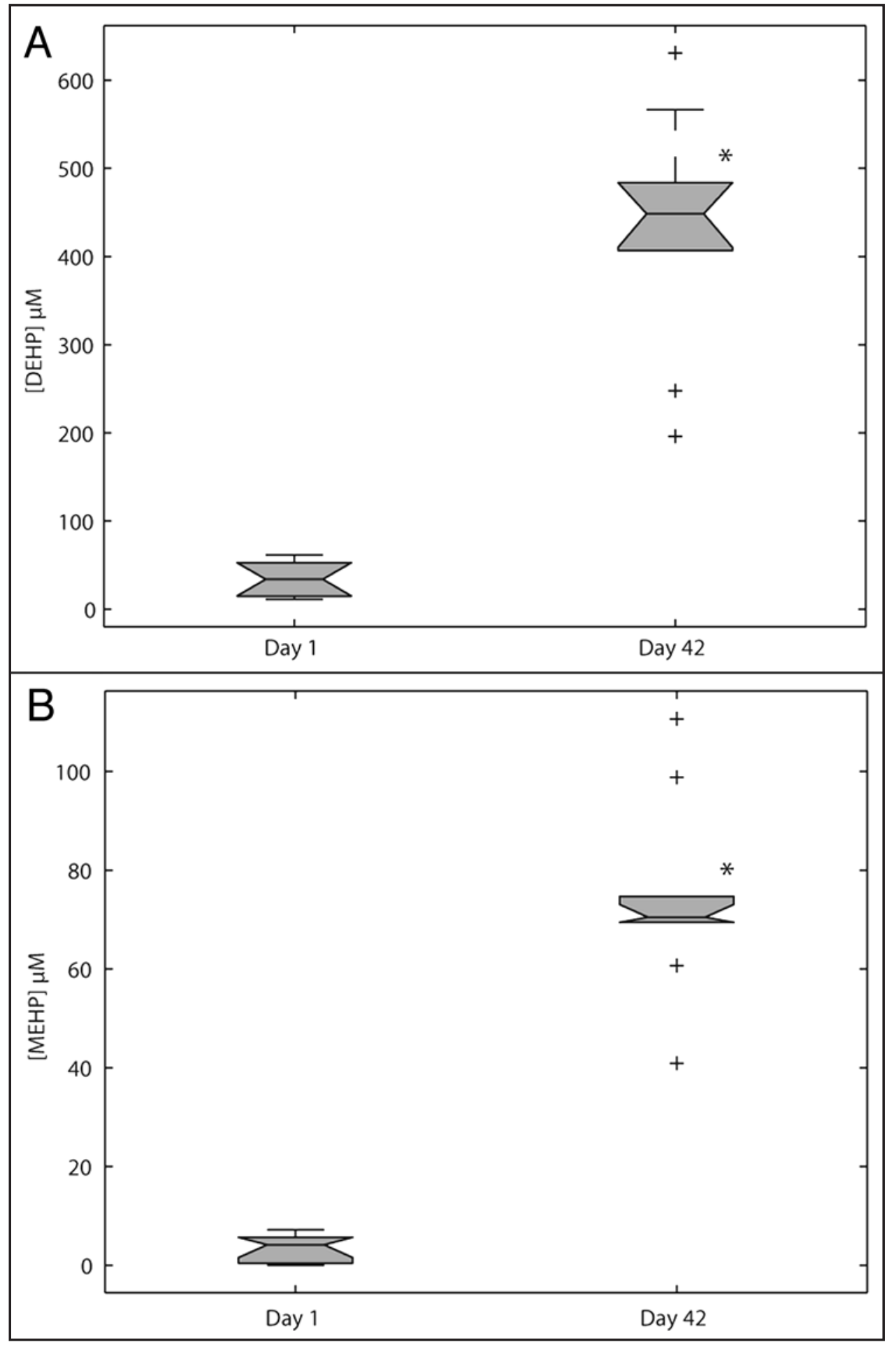

Figure 4. Total (A) DEHP and (B) MEHP in day 1 and day 42 supernatants collected from stored packed red blood cell (PRBC) units $(N=10)$. DEHP and MEHP levels were quantitated by liquid chromatography/mass spectrometry (LCMS) analysis. For each run, $10 \mu \mathrm{L}$ of sample was injected onto a YMC-Pack Protein-RP HPLC column (Waters, Milford, MA) heated to $50^{\circ} \mathrm{C}$. A 20 minute linear gradient from 10 to $40 \%$ solvent $B$ using water/0.1\% trifluoroacetic acid (solvent $A$ ) and acetonitrile/0.1\% trifluoroacetic acid (solvent B) was utilized with a flow rate of $1 \mathrm{~mL} / \mathrm{min}$. Data are expressed in micromolar $(\mu M)$ concentrations. An asterisk $\left({ }^{*}\right)$ designates significance where $p<0.05$ compared to day 1 supernatants (student t-test). Significant increases in DEHP and MEHP release from the PRBC bags were observed in the day 42 samples compared with the day 1 samples. 


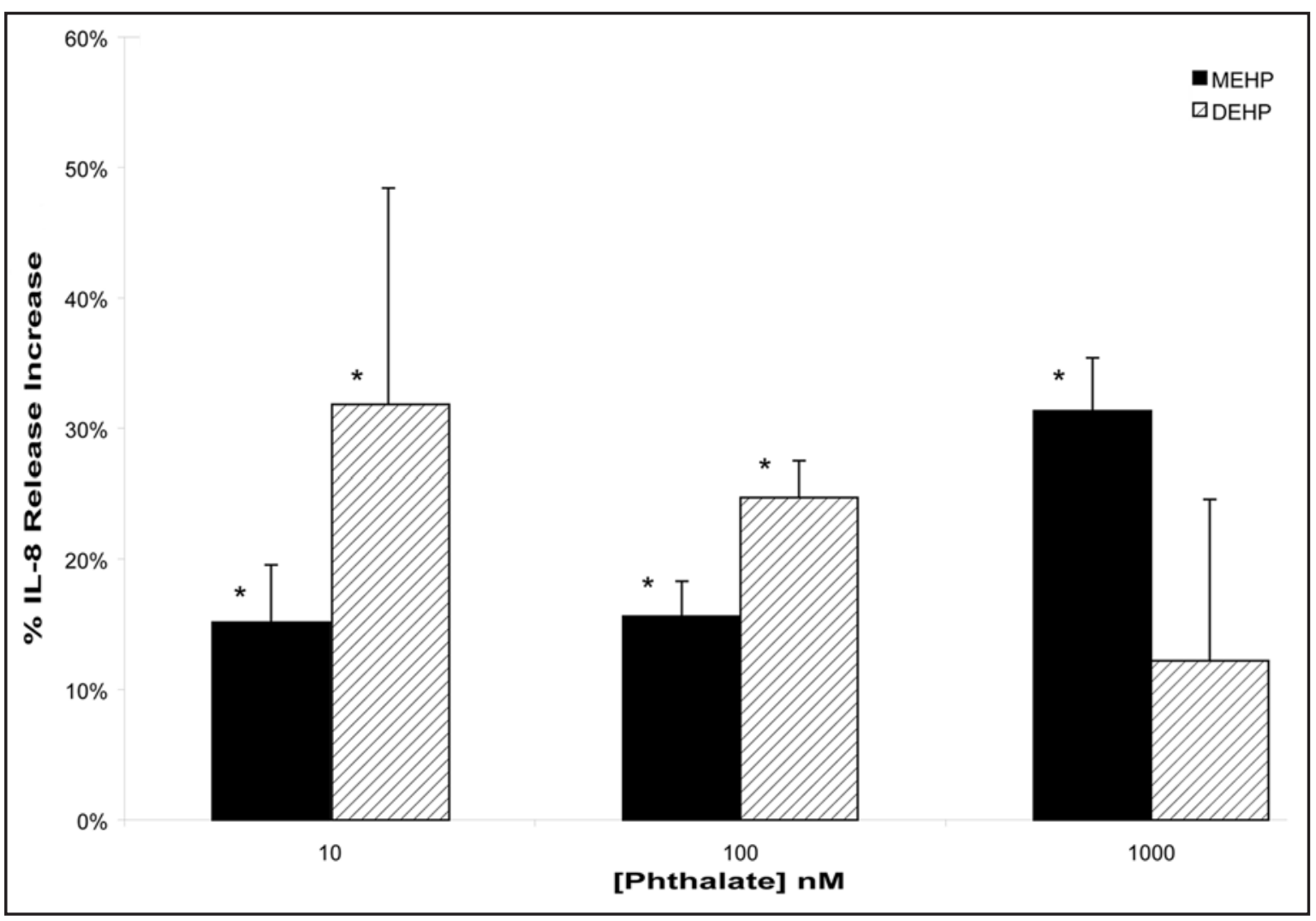

Figure 5. Effect of DEHP and MEHP on IL-8 release in HUVEC. HUVEC were grown to near confluency (>90\%) in 48-well plates in endothelial growth medium-2 (EGM-2) containing 2\% fetal calf serum, hydrocortisone, human fibroblast growth factor B, vascular endothelial growth factor, recombinant insulin-like growth factor-1, ascorbate, human epithelial growth factor, gentamycin and heparin. HUVEC were treated with media only (control), DEHP, or MEHP for 24 hours in duplicate. An IL-8 ELISA was performed on the 24 -hour supernatants. Data are expressed as \% IL- 8 release increase versus control wells. An asterisk (*) designates significance where $p<0.05$ compared to control wells (student t-test). At 10, 100 and 1,000 nM MEHP, a significant increase in IL-8 release was observed. At 10 and 100 nM DEHP, a significant increase in IL-8 release was observed. This indicates the potential proinflammatory capability of older units of stored packed red blood cells (PRBC).

\section{Materials and Methods}

Sample collection. Samples evaluated were obtained as part of a storage/leukoreduction study for PRBC. In brief, 10 whole blood units collected in citrate-phosphate-dextrose (CPD)/ AS-1 (ADSOL) buffer were obtained from healthy adult blood donors by standard techniques and stored for 24 hours at $4^{\circ} \mathrm{C}$. Packed red cells were prepared from whole blood by centrifugation of whole blood units at $4,200 \mathrm{rpm}$ for 5 minutes. AS- 1 buffer from a satellite bag was added to the main packed red cell bag according to standard procedure. Packed red cell units were subsequently stored for 42 days at $4-6^{\circ} \mathrm{C}$ in accordance with American Association of Blood Banks standards. Sample supernatants were collected from PRBC on storage days 1 and 42 by centrifugation at $1,000 \mathrm{~g}$ at $4^{\circ} \mathrm{C}$ for 10 minutes. A second centrifugation at $12,000 \mathrm{~g}$ was performed to remove membrane vesicles from stored cells. All samples were stored at $-80^{\circ} \mathrm{C}$ until further analysis.

LCMS analysis. Sample supernatants were analyzed by HPLC (Waters 2795 Separations Module, Milford, MA) coupled to positive electrospray ionization time of flight mass spectrometry
(+ESI-TOF MS, LCT, Micromass, UK) using a method described previously. ${ }^{24}$ Ten $\mu \mathrm{L}$ of each sample was injected onto a YMC-Pack Protein-RP HPLC column (Waters, Milford, MA) heated to $50^{\circ} \mathrm{C}$. A 20 minute linear gradient from 10 to $40 \%$ B using water $/ 0.1 \%$ trifluoroacetic acid (A) and AcN/0.1\% TFA (B) was utilized with a flow rate of $1 \mathrm{~mL} / \mathrm{min}$. The stock solutions for the DEHP $(1-1,000 \mu \mathrm{M})$ and MEHP $(1-100 \mu \mathrm{M})$ standards were initially dissolved in acetonitrile and then diluted in $1 \mathrm{X}$ phosphate-buffered saline (PBS) to working concentrations. Both DEHP and MEHP were obtained from Sigma-Aldrich (St. Louis, $\mathrm{MO})$. A student t-test was used to compare day 1 versus day 42 data to test for significant differences ( $p<0.05$, Microsoft Excel). All graphical data was generated using Matlab R14 (Mathworks, Natick, MA).

IL-8 release from HUVEC. The effect of DEHP and MEHP on IL-8 release from HUVEC was investigated. HUVEC cells (Cambrex, Walkersville, MD) were plated on 48-well plates in endothelial growth medium-2 (EGM-2) containing 2\% fetal calf serum (FCS), hydrocortisone, human fibroblast growth factor B, vascular endothelial growth factor, recombinant insulin-like growth factor-1, ascorbate, human epithelial growth factor, gentamycin 
and heparin (all obtained from Cambrex) and allowed to grow to $>90 \%$ confluency at $37^{\circ} \mathrm{C}, 5 \% \mathrm{CO}_{2}$. Prior to addition of peptides, all wells were rinsed once with EGM-2 medium which did not contain ascorbate and FCS, but did contain all the growth factors and was supplemented with $1 \mathrm{X}$ insulin-transferrin-sodium selenite (ITSS, Sigma, St. Louis, MO). The cells were treated in duplicate $(\mathrm{N}=3)$ with 10,100 or 1,000 nM DEHP or MEHP, and the cells were incubated for 24 hours at $37^{\circ} \mathrm{C}, 5 \% \mathrm{CO}_{2}$. After the 24-hour incubation, the supernatants were collected. The concentration of IL-8 in each supernatant was determined by ELISA (Pierce Biotechnology, Rockford, IL) using a microplate reader (Molecular Devices, M2 Microplate Reader, SpectraMax ${ }^{\circledR}$, Sunnyvale, CA) set at $450 \mathrm{~nm}$ with a $570 \mathrm{~nm}$ background subtraction. Data is expressed as \% change in IL-8 release compared to controls \pm standard deviation $(\mathrm{N}=3)$, and statistical differences from controls $(\mathrm{p}<0.05)$ were assessed using Student t-test (Microsoft Excel).

\section{References}

1. Latini G. Monitoring phthalate exposure in humans. Clin Chim Acta 2005; 361:20-9.

2. Wittassek M, Wiesmuller GA, Koch HM, Eckard R, Dobler L, Muller J, et al. Internal phthalate exposure over the last two decades-A retrospective human biomonitoring study. Int J Hyg Environ Health 2007; 210:319-33.

3. Hurst CH, Waxman DJ. Activation of PPAR $\alpha$ and PPAR $\gamma$ by environmental phthalate monoesters. Toxicol Sci 2003; 74:297-308.

4. Kavlock R, Barr D, Boekelheide K, Breslin W, Breysse P, Chapin R, et al. NTP-CERHR expert panel update on the reproductive and developmental toxicity of di(2-ethylhexyl) phthalate. Reprod Toxicol 2006; 22:291-399.

5. Jepsen KF, Abildtrup A, Larsen ST. Monophthalates promote IL-6 and IL-8 production in the human epithelial cell line A549. Toxicol In Vitro 2004; 18:265-9.

6. Larsen ST, Nielsen GD. The adjuvant effect of di-(2-ethylhexyl) phthalate is mediated through a PPAR $\alpha$-independent mechanism. Toxicol Lett 2007; 170:223-8.

7. Silliman CC, Voelkel NF, Allard JD, Elzi DJ, Tuder RM, Johnson JL, et al. Plasma and lipids from stored packed red blood cells cause acute lung injury in an animal model. J Clin Invest 1998; 101:1458-67.

8. Inoue K, Kawaguchi M, Yamanaka R, Higuchi T, Ito R, Saito K, et al. Evaluation and analysis of exposure levels of di(2-ethylhexyl) phthalate from blood bags. Clin Chim Acta 2005; 358:159-66.

9. Kambia K, Dine T, Azar R, Gressier B, Luyckx M, Brunet C. Comparative study of the leachability of di(2-ethylhexyl) phthalate and tri(2-ethylhexyl) trimellitate from haemodialysis tubing. Int J Pharm 2001; 229:139-46.

10. Pollack GM, Buchanan JF, Slaughter RL, Kohli RK, Shen DD. Circulating concentrations of di(2-ethylhexyl) phthalate and its de-esterified phthalic acid products following plasticizer exposure in patients receiving hemodialysis. Toxicol Appl Pharmacol 1985; 79:257-67.

11. Chu I, Villeneuve DC, Secours V, Franklin C, Rock G, Viau A. Metabolism and tissue distribution of mono-2-ethylhexyl phthalate in the rat. Drug Metab Dispos 1978; 6:146-9.

12. Griffiths WC, Camara PD, Saritelli A, Gentile J. The in vitro serum protein-binding characteristics of bis-(2-ethylhexyl) phthalate and its principal metabolite, mono-(2ethylhexyl) phthalate. Environ Health Perspect 1988; 77:151-6.

13. Rock G, Labow RS, Tocchi M. Distribution of di(2-ethylhexyl) phthalate and products in blood and blood components. Environ Health Perspect 1986; 65:309-16.

14. Dirven HA, van den Broek PH, Jongeneelen FJ. Effect of di(2-ethylhexyl)phthalate on enzyme activity levels in liver and serum of rats. Toxicology 1990; 65:199-207.

15. Rael LT, Bar-Or R, Aumann RM, Slone DS, Mains CW, Bar-Or D. Oxidation-reduction potential and paraoxonase-arylesterase activity in trauma patients. Biochem Biophys Res Commun 2007; 361:561-5.

16. Hillman RS, Ault KA, Rinder HM. Hematology in Clinical Practice: A Guide to Diagnosis and Management. New York: McGraw-Hill Professional 2005.

17. Rusyn I, Peters JM, Cunningham ML. Modes of action and species-specific effects of di-(2-ethylhexyl)phthalate in the liver. Crit Rev Toxicol 2006; 36:459-79.

18. Seo KW, Kim KB, Kim YJ, Choi JY, Lee KT, Choi KS. Comparison of oxidative stress and changes of xenobiotic metabolizing enzymes induced by phthalates in rats. Food Chem Toxicol 2004; 42:107-14.

19. Hong YC, Park EY, Park MS, Ko JA, Oh SY, Kim H, et al. Community level exposure to chemicals and oxidative stress in adult population. Toxicol Lett 2009; 184:139-44.

20. Jozkowicz A, Was H, Taha H, Kotlinowski J, Mleczko K, Cisowski J, et al. 15d-PGJ2 upregulates synthesis of IL-8 in endothelial cells through induction of oxidative stress. Antioxid Redox Signal 2008; 10:2035-46.
21. Rael LT, Rao NK, Thomas GW, Bar-Or R, Curtis CG, Bar-Or D. Combined cupricand cuprous-binding peptides are effective in preventing IL-8 release from endothelial cells and redox reactions. Biochem Biophys Res Commun 2007; 357:543-8.

22. Rael LT, Bar-Or R, Ambruso DR, Mains CW, Slone DS, Craun ML, et al. The effect of storage on the accumulation of oxidative biomarkers in donated packed red blood cells. J Trauma 2009; 66:76-81.

23. Rock G, Tocchi M, Ganz PR, Tackaberry ES. Incorporation of plasticizer into red cells during storage. Transfusion 1984; 24:493-8.

24. Bar-Or D, Bar-Or R, Rael LT, Gardner DK, Slone DS, Craun ML. Heterogeneity and oxidation status of commercial human albumin preparations in clinical use. Crit Care Med 2005; 33:1638-41. 


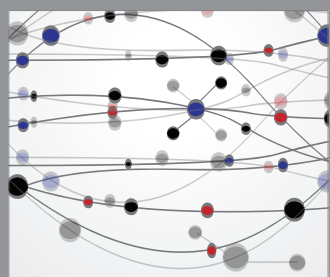

The Scientific World Journal
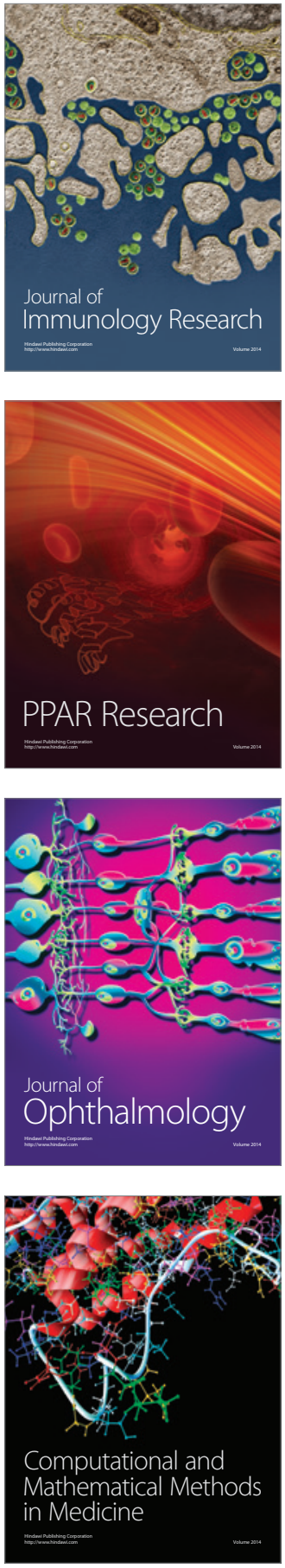

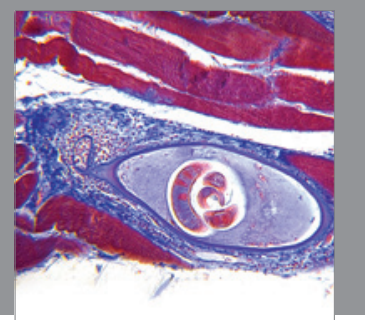

Gastroenterology

Research and Practice
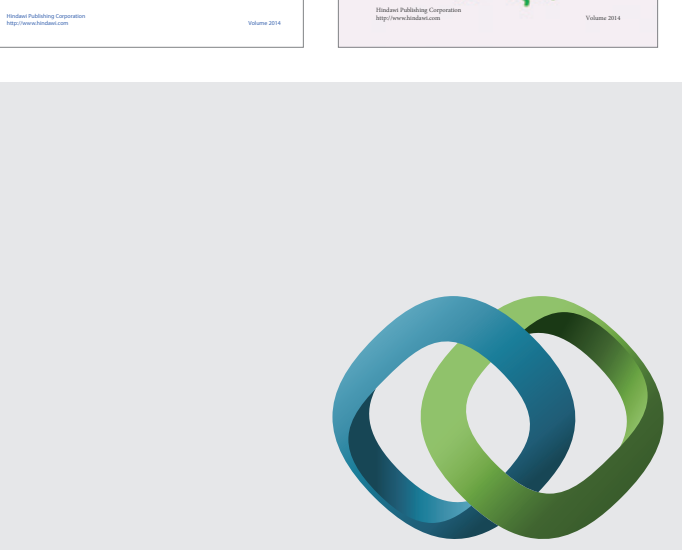

\section{Hindawi}

Submit your manuscripts at

http://www.hindawi.com
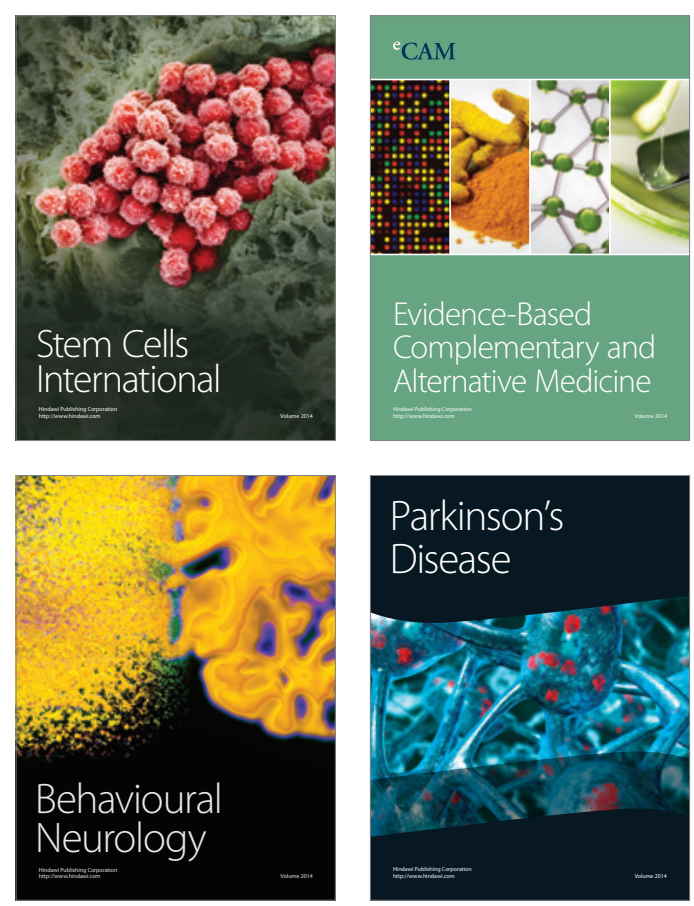

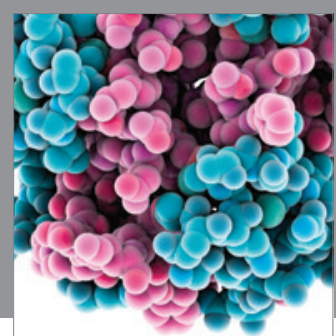

Journal of
Diabetes Research

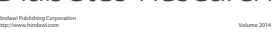

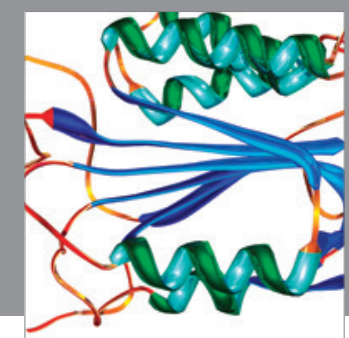

Disease Markers
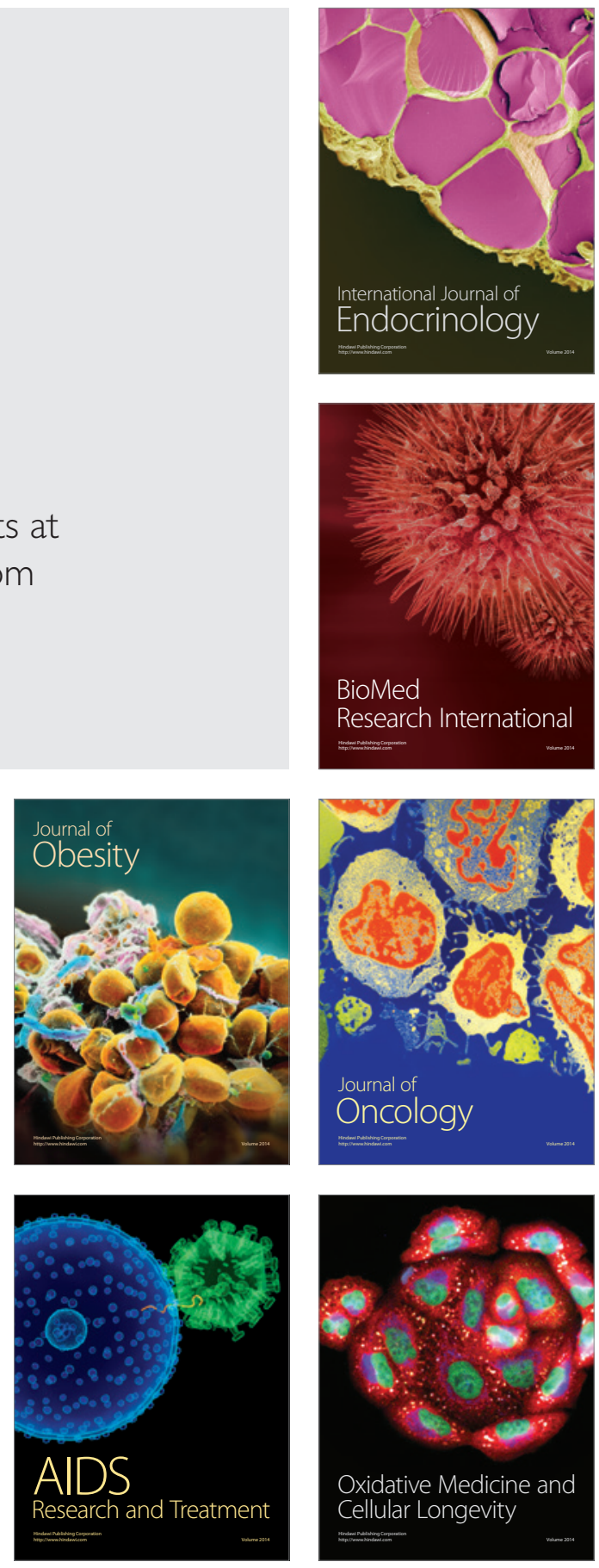